\title{
Identification of Species in Lycoris spp. from stigmatic exudate using electrochemical fingerprints
}

\author{
Weiting Ye $e^{1, \#}$, Yuhong Zheng ${ }^{2, \#}$, Pengcong Zhang ${ }^{3}$, Boyuan Fan ${ }^{1}$, Yongfang $\mathrm{Li}^{4}, \mathrm{Li} \mathrm{Fu}^{\mathrm{l}, *}$ \\ ${ }^{1}$ Key Laboratory of Novel Materials for Sensor of Zhejiang Province, College of Materials and \\ Environmental Engineering, Hangzhou Dianzi University, Hangzhou, 310018, PR. China \\ ${ }^{2}$ Institute of Botany, Jiangsu Province \& Chinese Academy of Sciences (Nanjing Botanical Garden \\ Mem. Sun Yat-Sen), Nanjing 210014, PR. China \\ ${ }^{3}$ Hangzhou Botanical Garden, Hangzhou, 310013, PR. China \\ ${ }^{4}$ Xiangshan County Agricultural Technology Extension Centre, Xiangshan, 315700, PR. China \\ *E-mail: fuli@hdu.edu.cn \\ \# These authors contributed equally.
}

Received: 8 July 2021 / Accepted: 16 August 2021 / Published: 10 September 2021

The use of electrochemical fingerprints for plant identification is an emerging application in biosensors. In this work, stigmatic exudate was collected from plants and the electrochemical fingerprints were taken. Lycoris anhuiensis, L. longituba, L. straminea, L. guangxiensis, L. haywardii, L. sprengeri, $L$. aurea, L. chejuensis, L. squamigera, L. qinlingensis, L. albiflora, L. radiata, L. incarnata and $L$. chinensis were collected for this purpose. Different electrochemical fingerprints were obtained in accordance with the differences of electrochemically active substances in the stigmatic exudate. These electrochemical fingerprint profiles can be adopted to construct different patterns of recognition strategies and further applied in the identification of species. In addition, since the electrochemical fingerprints of stigmatic exudate contain the overall information of electrochemically active substances, they can be adopted to study the relationship among different species. A phylogenetic tree was successfully constructed based on the data of electrochemical fingerprints. The deduced infrageneric relationship among species is more persuasive than that in classical chemotaxonomic studies. The results can rival those obtained from modern molecular taxonomic methods and provide insights for future genetic studies.

Keywords: Electroanalysis; Stigmatic exudate; Plant identification; Fingerprints; Biometrics

\section{$\underline{\text { FULL TEXT }}$}

(C) 2021 The Authors. Published by ESG (www.electrochemsci.org). This article is an open access article distributed under the terms and conditions of the Creative Commons Attribution license (http://creativecommons.org/licenses/by/4.0/). 